\title{
Glucosidase II exhibits similarity to the p53 tumor suppressor in regards to structure and behavior in response to stress signals: A potential novel cancer biomarker
}

\author{
BENJAMART SURADEJ $^{1}$, SUPANSA PATA ${ }^{2}$, WATCHARA KASINRERK ${ }^{2,3}$ and RATCHADA CRESSEY ${ }^{1}$ \\ Divisions of ${ }^{1}$ Clinical Chemistry and ${ }^{2}$ Clinical Immunology, Department of Medical Technology, Faculty of Associated \\ Medical Sciences, Chiang Mai University; ${ }^{3}$ Biomedical Technology Research Center, National Center for \\ Genetic Engineering and Biotechnology, National Science and Technology Development Agency at the \\ Faculty of Associated Medical Sciences, Chiang Mai University, Chiang Mai 50200, Thailand
}

Received June 26, 2013; Accepted August 7, 2013

DOI: $10.3892 / o r .2013 .2721$

\begin{abstract}
Early diagnosis of cancer is a key factor for the success of treatment. For this reason, identification of highly sensitive and specific novel tumor markers is urgently needed. In the present study, the CM5 polyclonal antibody (CM5 pAb) raised against p53 of mouse origin was used to identify p53 structurally related protein(s) that may also play an important role in promoting or preventing lung cancer. Western blot analysis was performed on tumor tissues and corresponding normal tissues obtained from lung cancer patients. CM5 pAb reacted with a human protein with an apparent molecular weight of $90 \mathrm{kDa}$ in the lung tumor tissue. The levels of this protein were greatly increased in 35 of the 37 (94.6\%) lung tumor samples assessed, with only minimal expression in the normal adjacent tissues. The $90-\mathrm{kDa}$ protein was immunoprecipitated by CM5 pAb and was subsequently identified by LC-MS/ MS to be glucosidase II, a key protein involved in the quality control mechanism of glycoprotein folding. An investigation of the response to genotoxic stress and endoplasmic reticulum (ER) stress using A549 human lung adenocarcinoma cells demonstrated that glucosidase II exhibited a similar pattern of response as the p53 tumor suppressor. Protein levels of both $\mathrm{p} 53$ and glucosidase II were increased in response to UV irradiation but decreased in response to tunicamycin-induced ER stress. In conclusion, we demonstrated that a polyclonal antibody raised against mouse p53 could cross-react with human glucosidase II, which was found to be frequently over-
\end{abstract}

Correspondence to: Dr Ratchada Cressey, Division of Clinical Chemistry, Department of Medical Technology, Faculty of Associated Medical Sciences, Chiang Mai University, Chiang Mai 50200, Thailand

E-mail: ratchada.cr@cmu.ac.th; ratchadacr@gmail.com

Key words: lung cancer, cancer biomarker, p53 tumor suppressor, ER stress, glucosidase II, genotoxic stress expressed in human lung tumor tissues and exhibited a stress response similar to p53. The high frequency of glucosidase II overexpression, which to the best of our knowledge has not been previously described, indicates its crucial roles in lung tumorigenesis and is thus a valuable biomarker for facilitating the screening and/or diagnosis of lung cancer. However, further investigations concerning its relationship to p53 and its roles in ER and genotoxic stress are warranted.

\section{Introduction}

The identification and characterization of the $\mathrm{p} 53$ protein has relied extensively on immunological methods. Antibodies directed against p53 protein have been valuable tools for investigating the structure-function relationship of wild-type and mutant p53 as well as other p53-related proteins such as p63 and p73. Monoclonal antibodies raised against Xenopus p53 have been demonstrated to cross-react with human p73 (1). The p53 proteins of both mouse and human origin are detectable with PAb240, reflecting a high sequence homology of the p53 protein between these two species. The epitope for the monoclonal PAb421 antibody (2) is also highly conserved between the human and the mouse (3).

The CM5 polyclonal antibody (CM5 pAb) from rabbits immunized with mouse wild-type p53 was originally developed for immunohistochemical analysis of mouse p53 expression (4). It has high affinity for mouse p53 and low affinity for human p53 and is also very useful in immunoblotting of mouse p53. CM5 pAb recognizes several antibody epitopes of the mouse p53 protein, including the epitope for PAb240 (3). Using an anti-mouse p53 CM5 pAb to perform immunoblotting of human lung tumor tissues, we unexpectedly found that the CM5 pAb cross-reacted with an unknown human protein with an apparent molecular weight of $90 \mathrm{kDa}$. The CM5-reactive protein was found to be overexpressed in human lung tumor tissues with only minimal expression in normal adjacent lung tissues. Therefore an investigation was carried out to identify and characterize this protein, which may play an important role in promoting or preventing lung cancer and could potentially be a novel lung tumor biomarker. 


\section{Materials and methods}

Patients and samples. Tissue samples were collected from patients who underwent curative surgery for lung cancer at Maharaj Nakorn Chiang Mai Hospital, Thailand. In each case, an adjacent normal tissue was also collected. These specimens were immediately placed in vials, frozen in embedded medium to preserve cell integrity, and stored at $-70^{\circ} \mathrm{C}$ until analyzed. The study was approved by the Ethics Committee of the faculty of Medicine, Chiang Mai University in compliance with the Helsinki Declaration (document no. 260/2005).

Western blot analysis. Frozen tissues were thawed, cut into small pieces and homogenized in an SDS lysis buffer [0.5 M Tris- $\mathrm{HCl} \mathrm{pH} 6.8,2 \%$ SDS (w/v) and 10\% glycerol (v/v)] containing a protease inhibitor cocktail (Complete, Mini, EDTA-free; Roche Applied Science, Indianapolis, IN, USA). The tissue homogenate was then centrifuged at $10,000 \mathrm{xg}$ for $15 \mathrm{~min}$ at $4^{\circ} \mathrm{C}$, after which the supernatant was removed and the protein concentration of the supernatant was determined using a BCA protein assay kit (Pierce Biotechnology, Inc., Rockford, IL, USA). Protein ( $30 \mu \mathrm{g})$ from the tumor tissue and adjacent normal tissue of each patient was resolved on $10 \%$ SDS polyacrylamide gels under reducing conditions and electrotransferred onto a PVDF membrane (Pierce Biotechnology, Inc.). The membrane was blocked with 5\% non-fat milk in TBS containing $0.05 \%$ Tween-20 (TBS-Tween) for $1 \mathrm{~h}$ before being incubated with polyclonal antibodies specific for mouse p53 (CM5 pAb, cat. no. NCL-p53-CM5p; Novocastra, Newcastle, UK) or monoclonal antibodies specific for glucosidase II (cat. no. sc-10774) or GRP-78 (cat. no. sc-13539), or GRP-94 (cat. no. sc-53929) (all from Santa Cruz Biotechnology, Inc., Santa Cruz, CA, USA) or human p53 (DO7; Novacastra) for $1 \mathrm{~h}$ at room temperature (RT). Bound antibodies were then detected with horseradish peroxidase (HRP)-conjugated goat anti-mouse IgG (cat. no. P 0448) or goat anti-rabbit IgG (cat. no. P 0447) (both from DakoCytomation, Carpinteria, CA, USA) for $1 \mathrm{~h}$ at RT, respectively. After extensive washing with TBS-Tween, immunoreactive protein was visualized with a chemiluminescence-based procedure using the ECL Plus detection kit according to the manufacturer's protocol (Amersham Pharmacia Biotech, Piscataway, NJ, USA).

Immunoprecipitation of the CM5 pAb-reactive protein from tumor cell lysate. Cell lysates from tumor tissues homogenized in SDS lysis buffer containing 2\% SDS were diluted with water in order to obtain $1 \mathrm{mg} / \mathrm{ml}$ of protein and $<0.05 \%(\mathrm{w} / \mathrm{v})$ final concentration of SDS. The diluted cell lysate was subjected to a preclearing step by incubating with suspended protein $G$ coated-agarose (cat. no. 20398; Pierce Protein Research Product) at $4^{\circ} \mathrm{C}$ for $45 \mathrm{~min}$. Subsequently, the precleared cell lysate was incubated with anti-mouse p53 CM5 pAb at $4^{\circ} \mathrm{C}$ for $4 \mathrm{~h}$ before adding precleared beads into the reaction tube and continued incubation at $4^{\circ} \mathrm{C}$ overnight. After extensive washing, the immunoprecipitated proteins were eluted from the bead particles by adding SDS lysis buffer containing 2 -mercaptoethanol and heating at $95^{\circ} \mathrm{C}$ for $5 \mathrm{~min}$. The eluted protein was then resolved through SDS-PAGE (10\% gel) and stained with Coomassie Blue.
Protein identification by mass spectrophotometry. After SDS-PAGE analysis of the immunoprecipitated CM5 pAbreactive protein, the protein band that migrated at $90 \mathrm{kDa}$ was excised and subjected to protein identification by mass spectrometry at the Genome Institute of Thailand (BIOTEC). The excised protein was analyzed by liquid chromatographytandem mass spectrometry (LC-MS/MS) using machine model Finningan LTQ linear ion trap mass spectrometer using a Finnigan Surveyor ${ }^{\mathrm{TM}}$ MS pump with a flow splitter HPLC system (Thermo Scientific) according to a previously described protocol by Mitprasat et al (5).

Characterization of the CM5 pAb-reactive human protein in response to $U V$ irradiation and tunicamycin-induced endoplasmic reticulum (ER) stress. Response of CM5 pAb-reactive protein to UV irradiation and ER stress was investigated using the A549 human lung adenocarcinoma epithelial cell line cultured in Dulbecco's modified Eagle's medium (DMEM) supplemented with $10 \%$ fetal bovine serum (FBS), $100 \mathrm{U} /$ $\mathrm{ml}$ penicillin and $100 \mu \mathrm{g} / \mathrm{ml}$ streptomycin. A549 $\left(5 \times 10^{5}\right)$ cells were seeded into a $100-\mathrm{mm}$ dish containing $10 \mathrm{ml}$ of DMEM and cultured at $37^{\circ} \mathrm{C}$ in a humidified incubator with $5 \% \mathrm{CO}_{2}$ overnight. The following morning, the culture medium was removed, and cells were irradiated with $\mathrm{UV}\left(15 \mathrm{~J} / \mathrm{m}^{2}\right)$ in order to induce DNA damage. After adding fresh medium, culture was continued at $37^{\circ} \mathrm{C}$ in $5 \% \mathrm{CO}_{2}$, and the cell lysate was prepared from UV-irradiated cells at different time points $(3,6,24$ and $36 \mathrm{~h}$ ). In order to induce ER stress, A549 cells were cultured in DMEM containing $3 \mu \mathrm{g} / \mathrm{ml}$ tunicamycin (cat. T7765; SigmaAldrich, St. Louis, MO, USA). Cell lysates were also prepared from tunicamycin-treated cells at different time points $(3,6$, 24 and $36 \mathrm{~h}$ ) and subjected to western blot analysis. Blots were probed with specific antibodies against human p53, glucosidase II, GRP (glucose-regulated protein)-78 and GRP-94 to characterize changes in the level of these proteins. Blots were also probed for GAPDH to confirm equal loading of protein.

\section{Results}

Overexpression of CM5-reactive protein in human lung tumor tissues. Anti-mouse p53 CM5 polyclonal antibody (CM5 pAb) was found to react with a human protein with an apparent molecular weight of $90 \mathrm{kDa}$. Of the 37 human lung tissue samples investigated, $35(94.6 \%)$ were found to have an increased level of this $90-\mathrm{kDa}$ protein in tumor tissues when compared to levels in the normal tissues. The overexpression of this unknown protein in tumor vs. normal adjacent lung tissues is shown in Fig. 1. It appeared that, in our detection system, human p53 was not recognizable by the CM5 pAb as no band at $53 \mathrm{kDa}$ was detected, although a number of tumor tissues showed a positive band with the DO7 anti-human p53 monoclonal antibody (Fig. 1B).

Purification and identification of the CM5 pAb-reactive protein. To identify the unknown protein recognized by CM5 pAb, immunoprecipitation was performed in order to isolate and purify this protein from the tumor cell lysate. The immunoprecipitated protein was resolved through $10 \%$ polyacrylamide gel. The protein band that migrated at $\sim 90 \mathrm{kDa}$ 
A Mw.
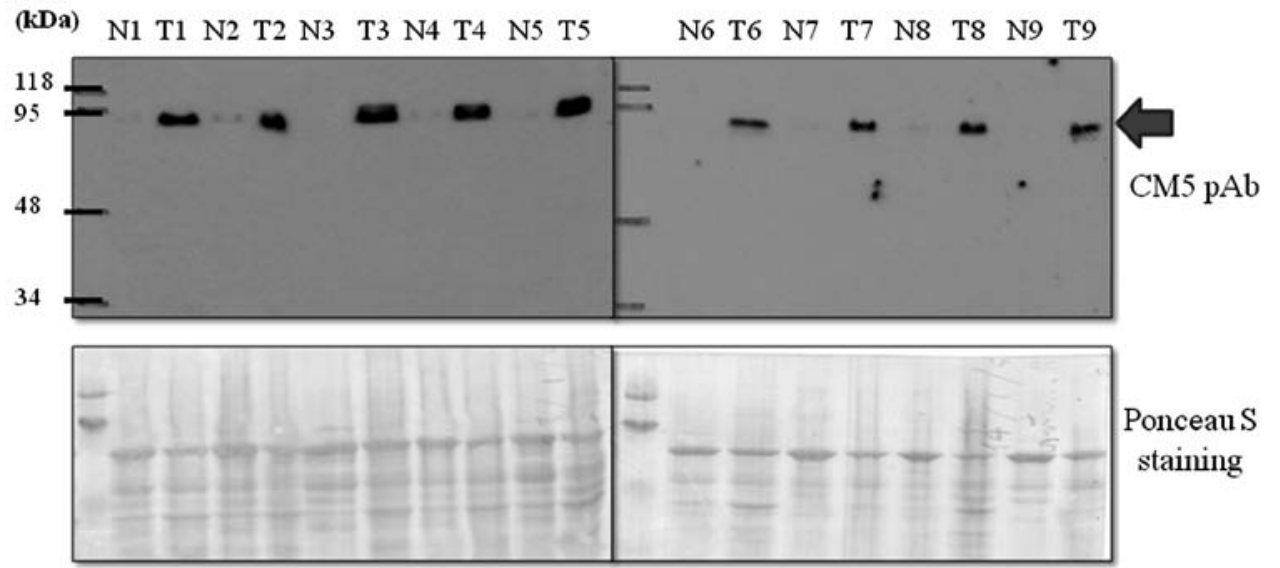

B Mw.

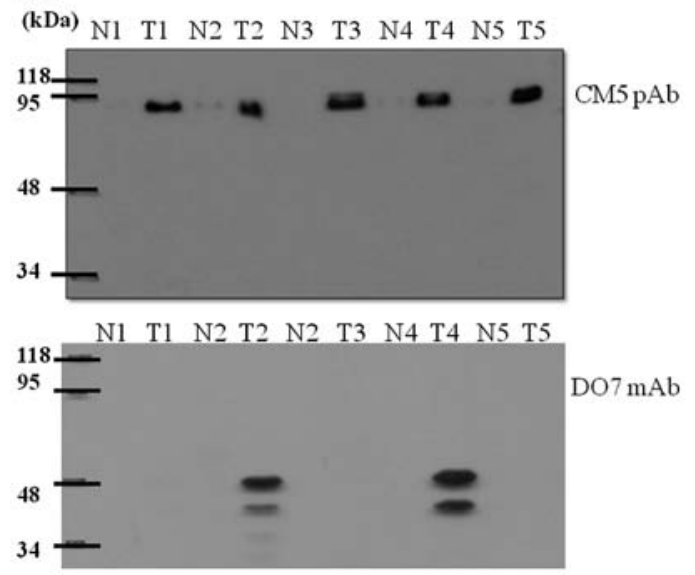

Figure 1. (A) Expression pattern of the CM5 pAb-reactive protein in lung tumor and corresponding normal tissues, and (B) the expression pattern with antihuman p53 (DO7) mAb. The amount of protein loaded into each lane is shown with Ponceau S staining; T, tumor tissue; N, normal tissue; CM5 pAb, CM5 polyclonal antibody.
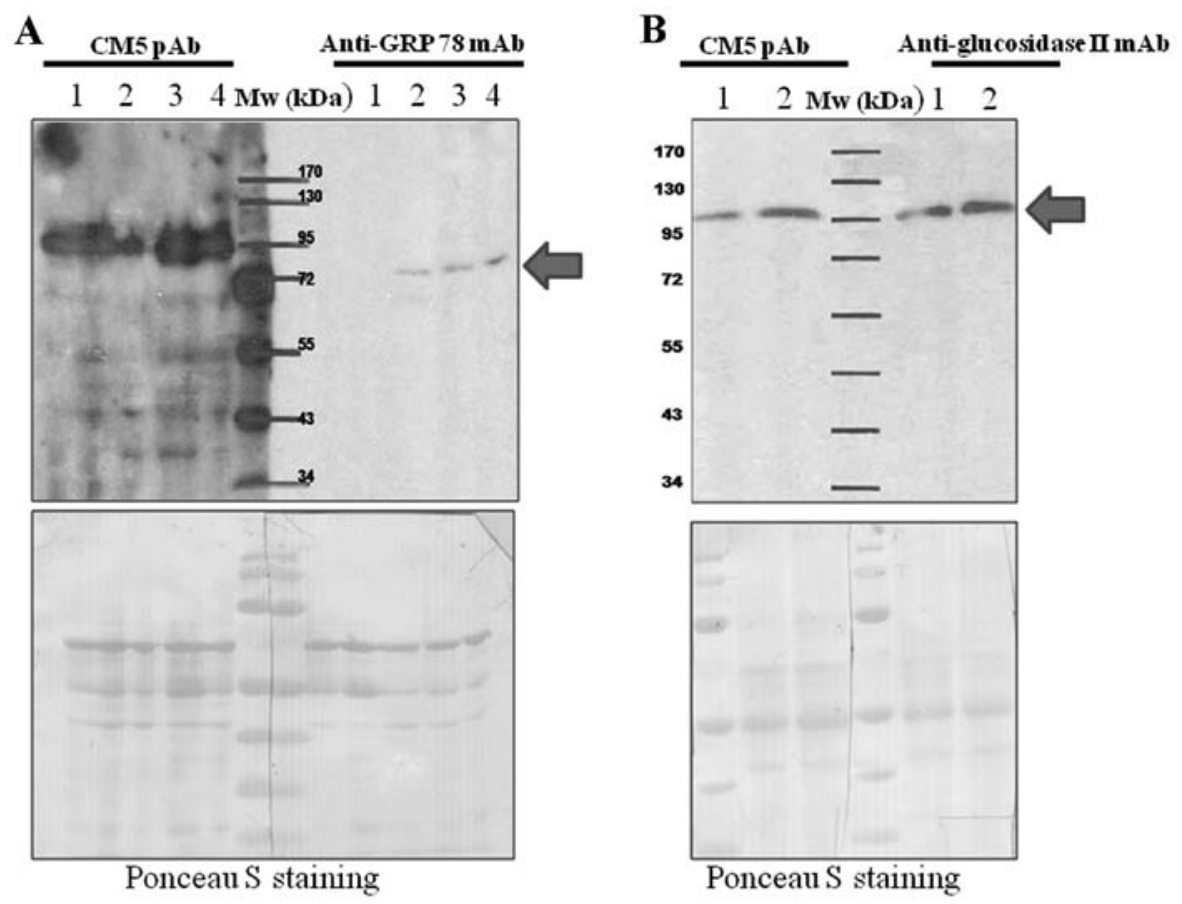

Figure 2. Comparison of the molecular weight of the CM5-reactive protein with (A) GRP78 and (B) glucosidase II. The number in each lane represents the tumor number of a patient sample known to overexpress the CM5 pAb-reactive protein. The amount of protein loaded into each lane is shown with Ponceau $\mathrm{S}$ staining. CM5 pAb, CM5 polyclonal antibody. 


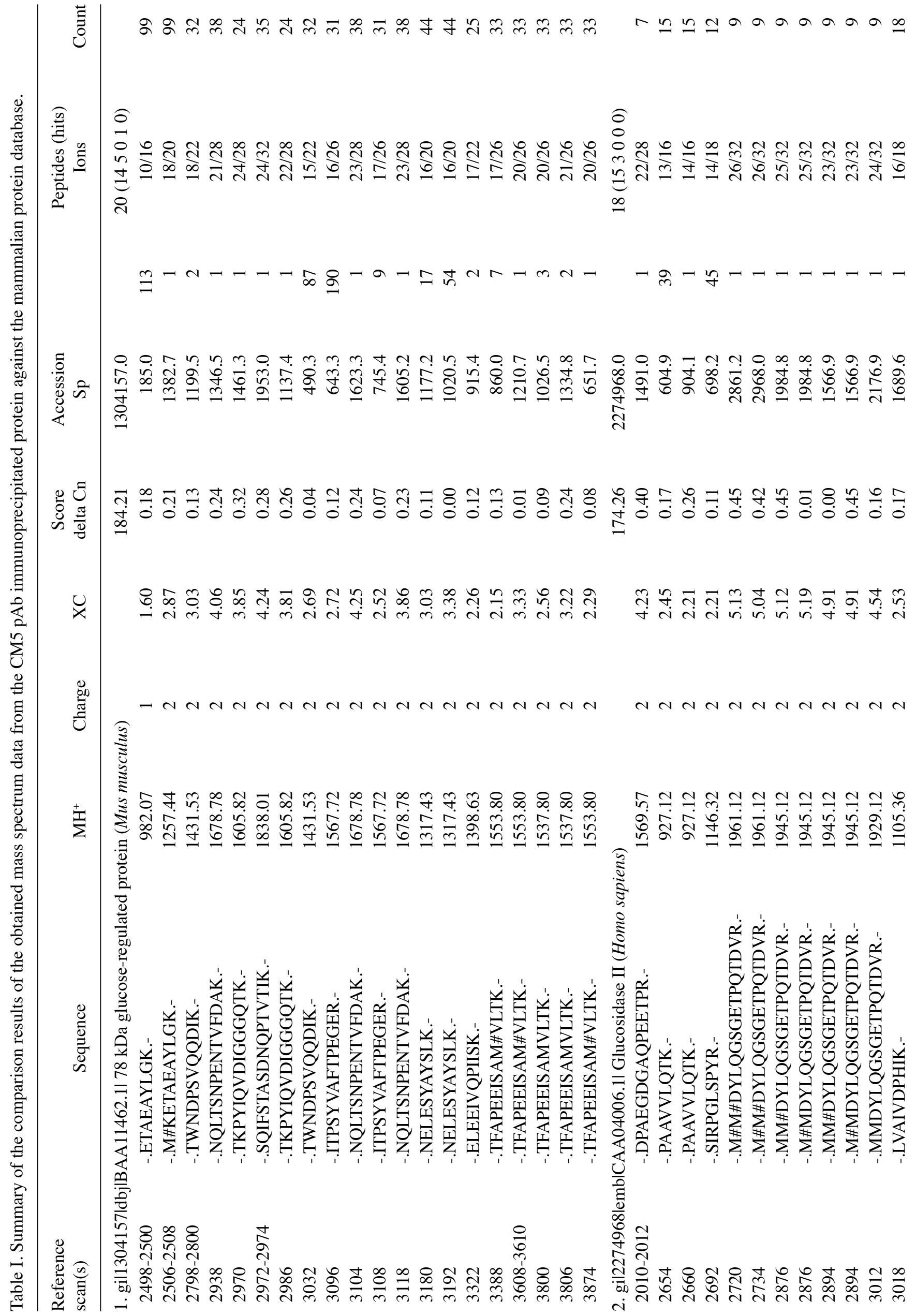




\begin{tabular}{|c|c|c|c|}
\hline$\vec{\Xi}$ & $\stackrel{\infty}{\longrightarrow} \sim r \underline{0}$ & 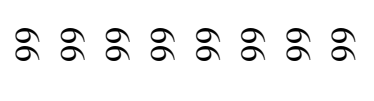 & 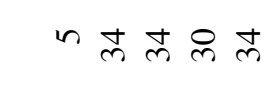 \\
\hline 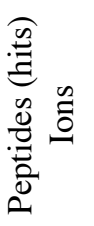 & 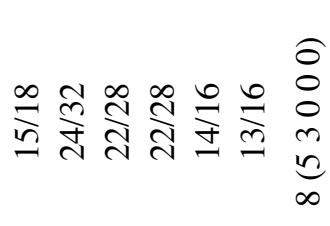 & 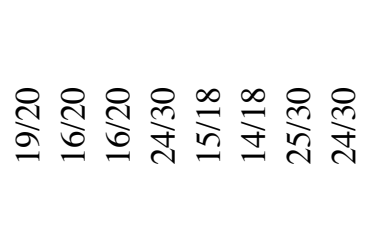 & 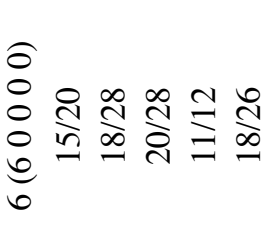 \\
\hline & $-\rightarrow-m \approx$ & $-\alpha \vartheta-r a-$ & $n--\vec{N} N$ \\
\hline 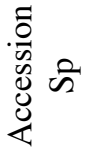 & 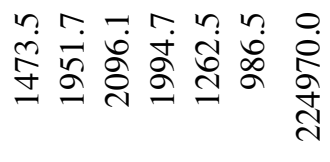 & 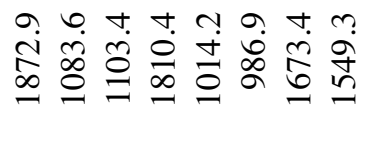 & 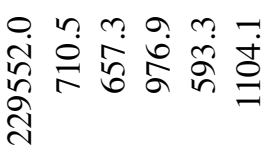 \\
\hline 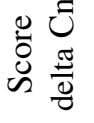 & 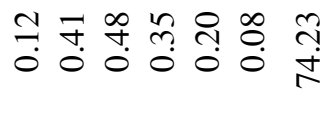 & 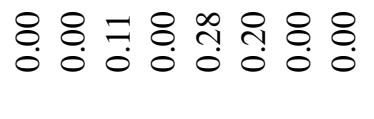 & 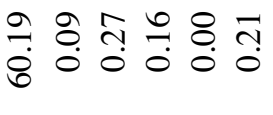 \\
\hline$\dot{x}$ & 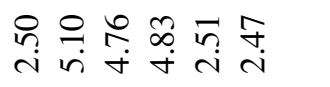 & 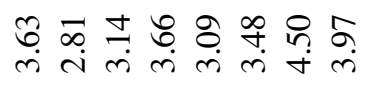 & 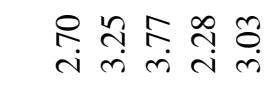 \\
\hline 惫 & 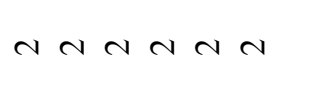 & $4 \sin \sin 4$ & $\mathrm{~N} N \mathrm{~N} N$ \\
\hline$\sum$ & 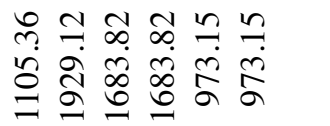 & 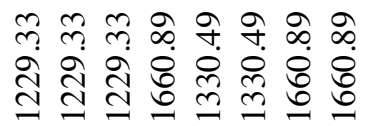 & 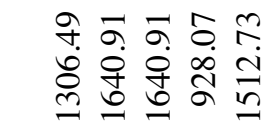 \\
\hline
\end{tabular}

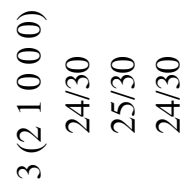

$\neg--$

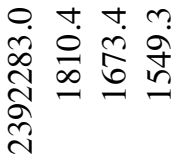

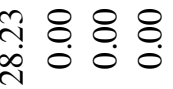

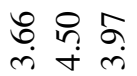

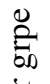

a n

焉

N $N$

के ळे के

용 8

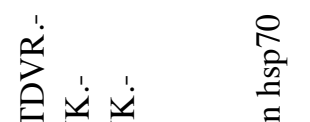

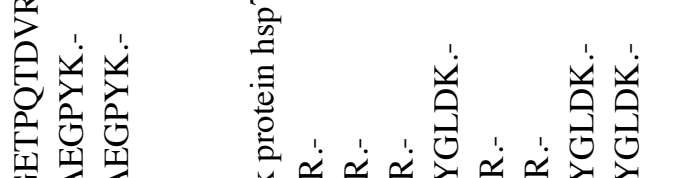

苛

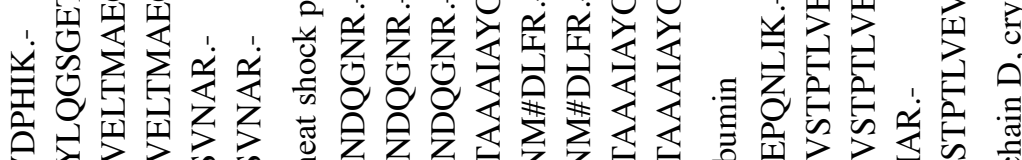

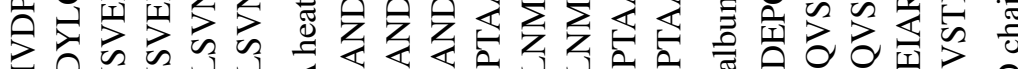

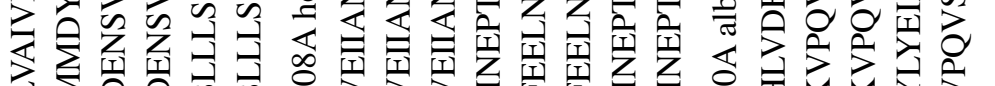

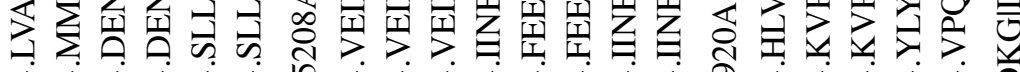




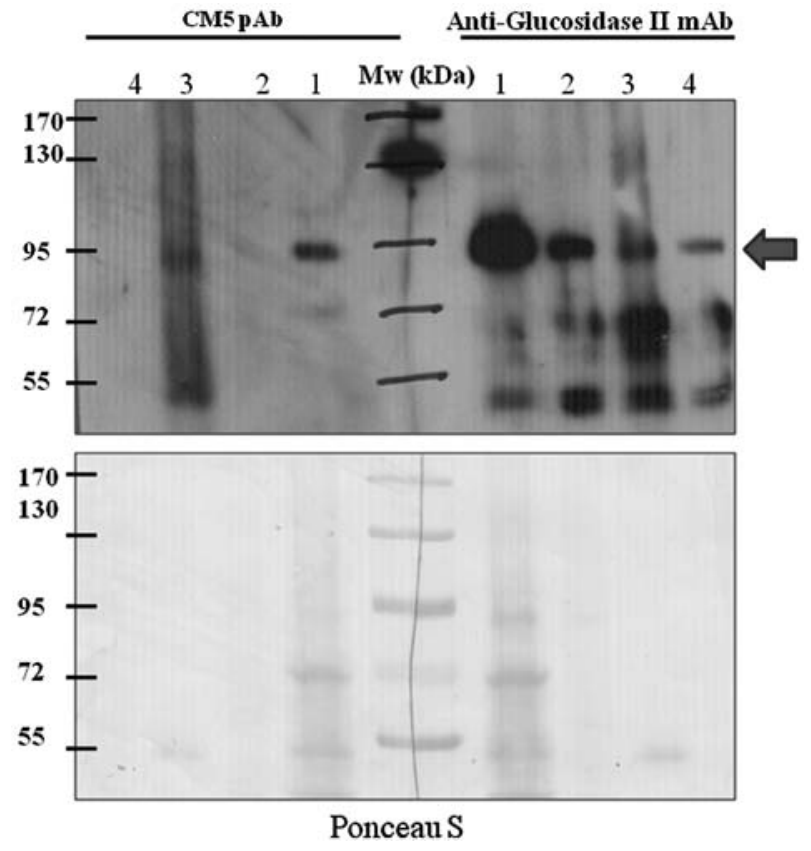

Figure 3. Western blot analysis showing the reactivity of the immunoprecipitated glucosidase II to CM5 pAb. Cell lysate from tumor tissues was diluted with water to obtain a protein concentration of $1 \mathrm{mg} / \mathrm{ml}$ and was immunoprecipitated with the antibody against glucosidase II. The imunoprecipitated protein was resolved through SDS-PAGE, transferred onto a PVDF membrane followed by immunodetection with the CM5 pAb and anti-glucosidase II mAb. Mw, molecular weight marker; lane 1, crude cell lysate from tumor tissue (30 $\mu \mathrm{g}$ of total protein); lane 2, diluted tumor cell lysate before immunoprecipitation; lane 3, immunoprecipitated protein; lane 4, diluted cell lysate after immunoprecipitation. The amount of protein loaded into each lane is shown with Ponceau S staining. CM5 pAb, CM5 polyclonal antibody.

was excised from the gel and subsequently subjected to protein identification using liquid chromatography-tandem mass spectrometry (LC-MS/MS). Using MS data searched against a mammarian protein database, the resulting mass spectra were identified as belonging to several candidate proteins (Table I). The most likely candidate proteins having the highest delta $\mathrm{Cn}$ score were mouse glucose-regulated protein, $78 \mathrm{kDa}$; GRP-78 (gi1304157, delta Cn score 184.21) and human ER glucosidase II (gi2274968, delta Cn score 174.26).

Verification of the protein identified by mass spectrophotometry. In order to verify the identification results of LC-MS/MS, protein molecular weight comparison and immunoprecipitation were performed. Whole cell lysate from various lung tumor tissues known to overexpress the CM5 pAb-reactive protein was resolved through $10 \%$ polyacrylamide gel, and separated proteins were transfered onto a PVDF membrane. The blots were cut in half and immunodetected with either CM5 pAb and anti-GRP-78 or anti-glucosidase II. As shown in Fig. 2, the unknown protein recognized by CM5 pAb had an apparent molecular weight similar to glucosidase II but not GRP-78. Therefore, immunoprecipitation was performed using anti-glucosidase II mAb. The immunoprecipitated protein was resolved through SDS-PAGE and subjected to immunodetection with CM5 pAb. The immunoprecipitated protein was recognizable by anti-glucosidase II $\mathrm{mAb}$ thus indicating the successful immunoprecipitation process. Notably, the immunoprecipitated protein was also recognized by CM5 pAb (Fig. 3). In addition, the results of the western blot analysis demonstrated an identical expression pattern between the proteins recognized by CM5 pAb and anti-glucosidase II $\mathrm{mAb}$ in each lung tumor tissue tested (representative western blot results are shown in Fig. 4).

Expression of glucosidase II in response to ER stress and UV irradiation. The A549 human lung adenocarcinoma epithelial cell line was used as a cell model to investigate the expression pattern of glucosidase II in response to UV-irradiation and tunicamycin-induced ER stress in comparison to $\mathrm{p} 53$. The
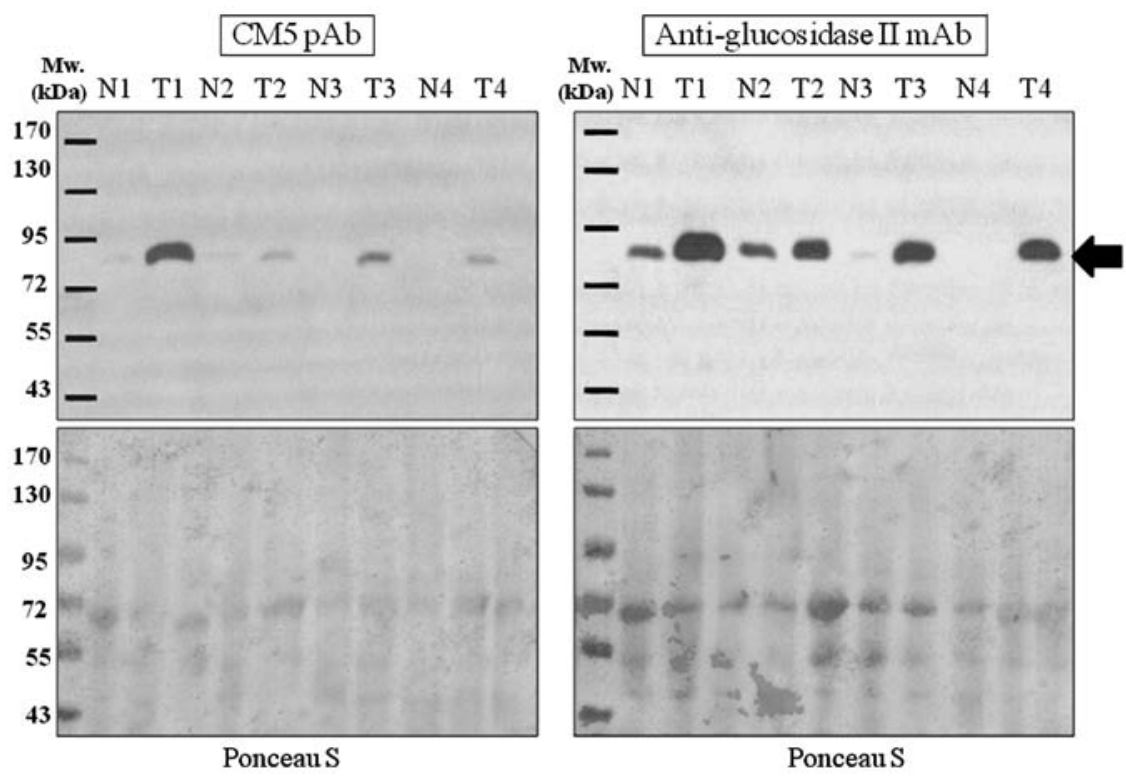

Figure 4. Results of the western blot analysis showing similarity in the expression of CM5-reactive protein and glucosidase II in lung tumor and corresponding normal tissues. The amount of protein loaded into each lane is shown with Ponceau S staining; T, tumor tissue; N, normal tissue; CM5 pAb, CM5 polyclonal antibody. 
A

Time after UV irradiation (h)

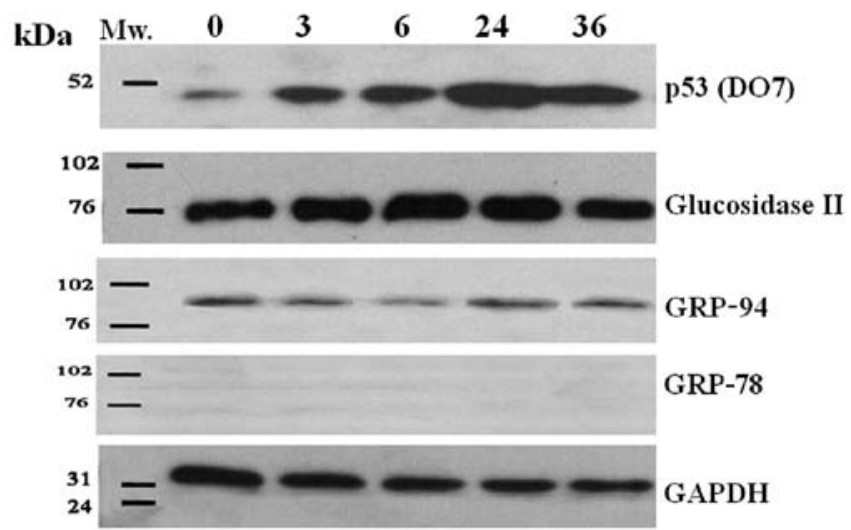

B

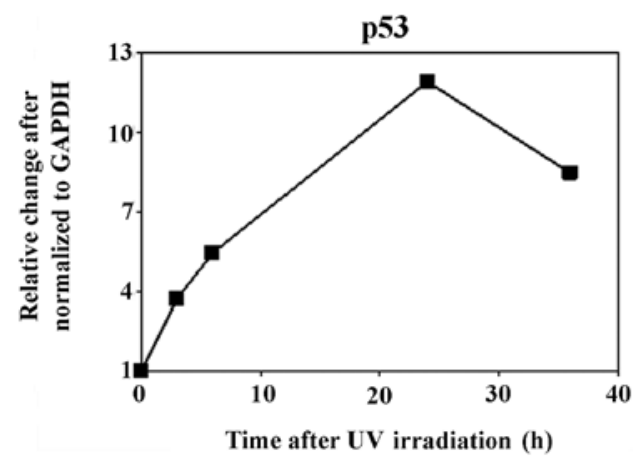

Glucosidase II

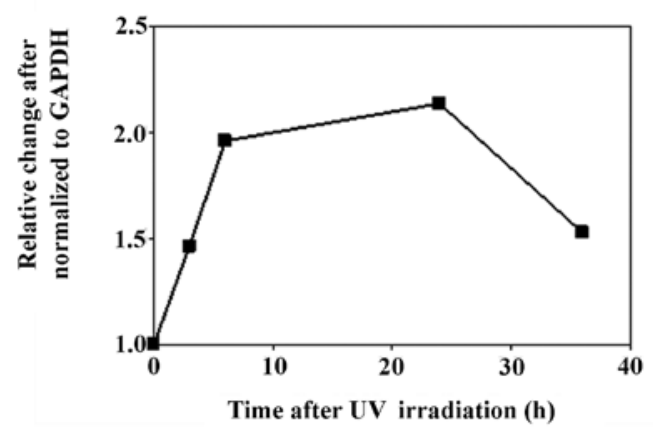

Figure 5. (A) Western blot analysis and (B) quantification of values showing changes in levels of various proteins in A549 lung adenocarcinoma cells at various time points following UV irradiation. Western blot bands were quantified using Quantity One (Bio-Rad Laboratories). Each band was normalized to corresponding values of GAPDH. The normalized values for each time point were then compared to the corresponding band of the untreated sample $(0 \mathrm{~h})$.

whole cell lysate was prepared at different time points $(0,3$, 6,24 and $36 \mathrm{~h}$ ) following treatment. As shown in Fig. 5A, the protein levels of both p53 and glucosidase II were increased in the UV-irradiated cells. The induction appeared as rapidly as $3 \mathrm{~h}$ after treatment and continued to increase even after $24 \mathrm{~h}$. The maximum induction was observed at $24 \mathrm{~h}$ for both p53 and glucosidase II (Fig. 5B). In contrast, levels of both p53 and glucosidase II were found to be suppressed in the tunicamycin-treated cells and the maximum reduction appeared at $6 \mathrm{~h}$ after treatment for both p53 and glucosidase II (Fig. 6A and B).
A Time after tunicamycin treatment (h)

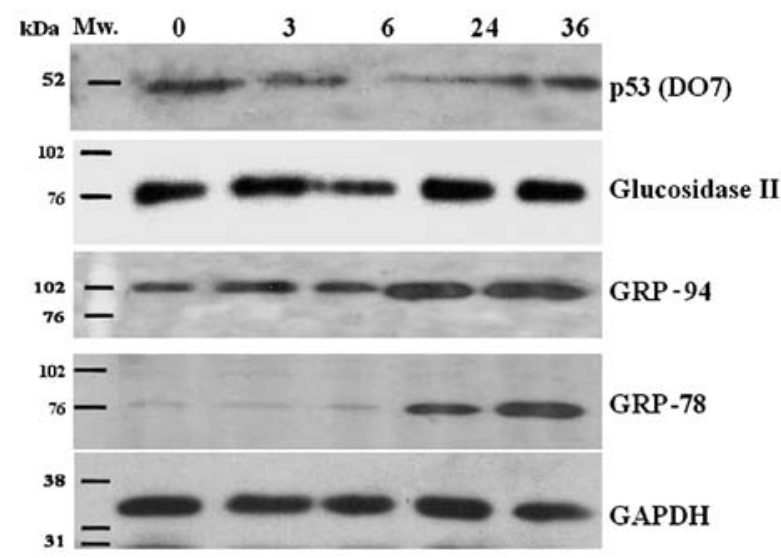

B

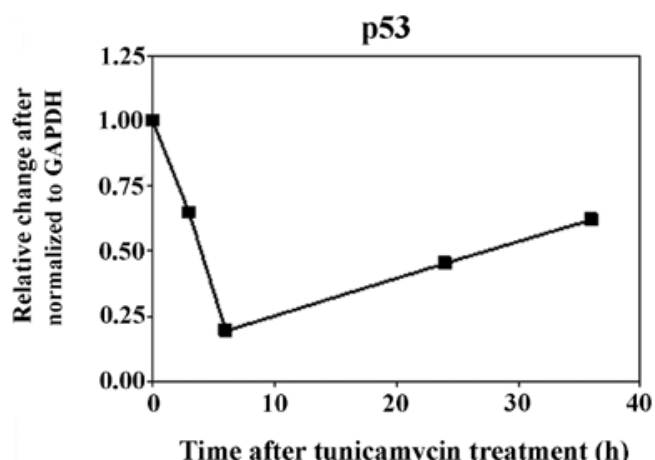

Glucosidase II

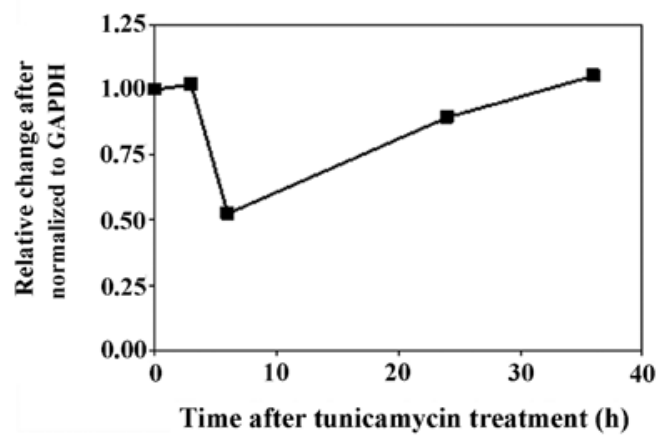

Figure 6. (A) Western blot analysis and (B) quantification of values showing changes in the levels of various proteins in the A549 lung adenocarcinoma cells at various time points following tunicamycin treatment. Western blot bands were quantified using Quantity One (Bio-Rad Laboratories). Each band was normalized to corresponding values of GAPDH. The normalized values for each time point were then compared to the corresponding band of the untreated sample $(0 \mathrm{~h})$.

\section{Discussion}

Using the CM5 anti-mouse p53 polyclonal antibody to performed western blot analysis of human lung tumor tissues, we fortuitously discovered that the protein level of glucosidase II was greatly increased in a high proportion of lung tumor tissues, while the corresponding normal adjacent tissues showed barely detectable expression levels. Glucosidase II plays a key role in the processing of $\mathrm{N}$-linked oligosaccharide chains of glycoprotein and is involved in the quality control mechanism of glycoprotein folding (6). However, to the best of our 
knowledge, overexpression of glucosidase II in tumor tissues has not been previously described. ER is the site of folding for protein destined for different compartments of the cell. As the newly synthesized proteins enter the ER lumen, they are covalently modified by the addition of a pre-assembled oligosacharide composed of $2 \mathrm{~N}$-acetylglucosamines, 9 mannoses and 3 glucoses. Glycosylation of the newly synthesized protein is believed to aid in the increasing hydrophilicity of the un-structured proteins. Subsequently, the glycosylated protein chains enter a glycoprotein-dedicated chaperone system comprising calnexin and calreticulin (7). Access to the calnexin/calreticulin system requires removal of the two outermost glucose residues by the sequential action of glucosidase I and II, respectively (8). Removal of the third glucose is also mediated through glucosidase II action, which is required to dissociate folding substrates from calnexin and the release of the native proteins from the ER and transport to their final destination. However, if the protein is improperly folded, the folding sensor UDP-glucose:glycoprotein glucosyltransferase 1 (UGT1) will add back a terminal glucose to promote re-association of the non-native protein and calnexin, thus prolonging their time in the ER folding environment. Cycles of de-/re-glycosylation may be extended until the protein released from calnexin fufills the quality requirement (reviewed in ref. 6). Therefore, balance of glucosidase II and UGT1 activity is crucial in order to maintain protein quality control of the ER.

Disturbance in the folding capacity of the ER caused by a variety of endogenous and exogenous stimulants could initiate a cellular stress condition known as ER stress. ER stress is initially induced to re-establish ER homeostasis through the activation of an integrated intracellular signal transduction termed the unfolded protein response (UPR). However, when ER stress is too severe or prolonged, the pro-survival function of the UPR turns into a toxic signal, which is predominantly executed by mitochondrial apoptosis (9). Since glucosidase II plays a major role in the release of proteins from the ER folding system, cells with overexpression of glucosidase II may be impervious to ER stress. Even overloaded with misfolded proteins, UPR and apoptosis may not be triggered in glucosidase II-overexpressed cells. In agreement with this hypothesis, inhibition of glucosidase II has been reported to reduce proliferation and induce apoptosis $(10,11)$ of tumor cells.

There is increasing evidence that the UPR is compromised in a large variety of human tumors (reviewed in ref. 12). More importantly, several recent studies have demonstrated that interfering with the activation of different arms of the UPR (i.e., PERK-eIF2 $\alpha$-ATF4 axis) or altering the levels of the ER molecular chaperone GRP-78/BIP (a master regulator of ER function and the UPR) can inhibit tumor growth (13-15). This evidence indicates that not only the UPR is compromised in tumors but that it contributes to survival or growth of the cancer cells. Therefore, inhibitors of glucosidase II could also be regarded as potential anticancer agents $(16,17)$.

The discovery of the induction of glucosidase II protein in lung tumor tissues through the use of the antibody against p53 indicates their structure similarity, and prompted us to elucidate the connection between these two proteins. Although our findings remain to be confirmed in different cell lines and primary cultures from patients, we showed that the pattern of changes in the protein levels of p53 and glucosidase II in response to ER stress and genotoxic stress was similar in A549 cells. The p53 tumor-suppressor protein becomes stabilized and activated in response to a number of stressful stimuli, i.e., hypoxia, nucleotide depletion or oncogene activation (18). The main form of stress that activates p53 is genotoxic stress (19), which if left unchecked can lead to loss of genomic integrity and tumorigenesis. Activation of p53 allows it to carry out its function as a tumor suppressor protein through a number of controlling endpoints i.e., cell cycle arrest or apoptosis (20). However, it was not recognized until recently that ER stress also plays a pivotal role in modulating p53 activity. The protein level (21) and function (22) of p53 have been reported to be reduced or suppressed in response to ER stress. This inhibition is believed to help ensure that p53 activation is restricted to agents that induce genotoxic stress (23). However, if the ER stress is not properly responded or if the UPR is compromised by an altered level of regulator proteins, i.e. glucosidase II, this may lead to the overproduction of misfolded proteins which may be oncogenic and together with the suppression of p53 function, the cell may finally become cancerous. The fact that both p53 and glucosidase II respond to ER stress and genotoxicity in a similar fashion indicates the possible connection between the two proteins; our findings warrant further investigation.

In conclusion, we demonstrated that human glucosidase II protein was frequently overexpressed in human lung tumor tissues. The high frequency of glucosidase II overexpression, which to the best of our knowledge has not been previously described, indicates the crucial role of glucosidase II in lung tumorigenesis and its potential value as a biomarker for aiding the screening and/or diagnosis of lung cancer. For example, a highly sensitive glucosidase assay system could be developed to measure glucosidase enzymatic activity from non-invasive samples i.e., exhaled breath condensate (EBC) or pleural effusion, in order to screen for lung cancer. Further investigation concerning the underlying mechanism of the protein induction and its relationship with p53, genotoxic stress and ER stress are warranted.

\section{Acknowledgements}

This study was financially sponsored by the Research Chair Grant, National Sciences and Technology Development Agency (Thailand), the National Research Council of Thailand (NRCT) and the Faculty of Associated Medical Sciences, Chiang Mai University. We thank Dr Tim R. Cressey, Harvard School of Public Health and Chiang Mai University for his review and editing of the manuscript.

\section{References}

1. Le Bras M, Delattre V, Bensaad K, Blandino G and Soussi T: Monoclonal antibodies raised against Xenopus p53 interact with human p73. Oncogene 21: 1304-1308, 2002.

2. Harlow E, Crawford L, Pim D and Williamson N: Monoclonal antibodies specific for simian virus 40 tumor antigens. J Virol 39: 861-869, 1981.

3. Lane DP, Stephen CW, Midgley CA, et al: Epitope analysis of the murine p53 tumour suppressor protein. Oncogene 12: 2461-2466, 1996.

4. Midgley CA, Owens B, Briscoe CV, Thomas DB, Lane DP and Hall PA: Coupling between gamma irradiation, p53 induction and the apoptotic response depends upon cell type in vivo. J Cell Sci 108: 1843-1848, 1995. 
5. Mitprasat M, Roytrakul S, Jiemsup S, Boonseng $\mathrm{O}$ and Yokthongwattana K: Leaf proteomic analysis in cassava (Manihot esculenta, Crantz) during plant development, from planting of stem cutting to storage root formation. Planta 233: 1209-1221, 2011.

6. Ruddock LW and Molinari M: N-glycan processing in ER quality control. J Cell Sci 119: 4373-4380, 2006.

7. Ellgaard L, Molinari M and Helenius A: Setting the standards: quality control in the secretory pathway. Science 286: 1882-1888, 1999.

8. Wang N, Daniels R and Hebert DN: The cotranslational maturation of the type I membrane glycoprotein tyrosinase: the heat shock protein 70 system hands off to the lectin-based chaperone system. Mol Biol Cell 16: 3740-3752, 2005.

9. Lee AS and Hendershot LM: ER stress and cancer. Cancer Biol Ther 5: 721-722, 2006.

10. Magyar JE, Gamberucci A, Konta L, et al: Endoplasmic reticulum stress underlying the pro-apoptotic effect of epigallocatechin gallate in mouse hepatoma cells. Int J Biochem Cell Biol 41: 694-700, 2009.

11. Gamberucci A, Konta L, Colucci A, et al: Green tea flavonols inhibit glucosidase II. Biochem Pharmacol 72: 640-646, 2006.

12. Ranganathan AC, Adam AP, Zhang L and Aguirre-Ghiso JA: Tumor cell dormancy induced by p38SAPK and ER-stress signaling: an adaptive advantage for metastatic cells? Cancer Biol Ther 5: 729-735, 2006.

13. Cho HY, Thomas S, Golden EB, et al: Enhanced killing of chemo-resistant breast cancer cells via controlled aggravation of ER stress. Cancer Lett 282: 87-97, 2009.
14. Jakobsen CH, Størvold GL, Bremseth H, et al: DHA induces ER stress and growth arrest in human colon cancer cells: associations with cholesterol and calcium homeostasis. J Lipid Res 49: 2089-2100, 2008

15. Zhang LJ, Chen S, Wu P, et al: Inhibition of MEK blocks GRP78 up-regulation and enhances apoptosis induced by ER stress in gastric cancer cells. Cancer Lett 274: 40-46, 2009.

16. Elbein AD: Glycosidase inhibitors as antiviral and/or antitumor agents. Semin Cell Biol 2: 309-317, 1991.

17. Elbein AD: Glycosidase inhibitors: inhibitors of N-linked oligosaccharide processing. FASEB J 5: 3055-3063, 1991.

18. Horn HF and Vousden KH: Coping with stress: multiple ways to activate p53. Oncogene 26: 1306-1316, 2007.

19. Colman MS, Afshari CA and Barrett JC: Regulation of p53 stability and activity in response to genotoxic stress. Mutat Res 462: 179-188, 2000

20. Amundson SA, Myers TG and Fornace AJ Jr: Roles for p53 in growth arrest and apoptosis: putting on the brakes after genotoxic stress. Oncogene 17: 3287-3299, 1998.

21. Pluquet O, Qu LK, Baltzis D and Koromilas AE: Endoplasmic reticulum stress accelerates p53 degradation by the cooperative actions of $\mathrm{Hdm} 2$ and glycogen synthase kinase $3 \beta$. Mol Cell Biol 25: 9392-9405, 2005

22. Qu L and Koromilas AE: Control of tumor suppressor p53 function by endoplasmic reticulum stress. Cell Cycle 3: 567-570, 2004.

23. Stavridi ES and Halazonetis TD: p53 and stress in the ER. Genes Dev 18: 241-244, 2004. 